\title{
KECK SPECTROPOLARIMETRY OF HIGH REDSHIFT RADIO GALAXIES
}

\author{
A. CIMATTI*, A. DEY AND W. VAN BREUGEL \\ Institute of Geophysics and Planetary Physics, LLNL - 7000 \\ East Ave, P.O. Box 808, L-413, Livermore, CA 94550, USA \\ *On leave from Osservatorio Astrofisico di Arcetri, Italy \\ R. ANTONUCCI
}

Physics Dept., University of California at Santa Barbara, CA 93106, USA

AND

H. SPINRAD

Astronomy Dept., University of California at Berkeley - 601 Campbell Hall, Berkeley, CA 94720, USA

\section{Introduction}

High redshift radio galaxies ( $\mathrm{H} z \mathrm{RGs}$ ) are observable up to cosmological distances competitive with the most distant quasars. However, before using them as probes of galaxy evolution, it is crucial to separate the stellar and non-stellar components. One of the most striking properties of $\mathrm{H} z \mathrm{RG}$ is the alignment of the UV continuum with the axis of the radio source (alignment effect; McCarthy et al. 1987). However, the relative importance of the stellar and non-stellar radiation to the alignment effect is still unknown, although a significant fraction is recognized to come from scattering of anisotropic radiation emitted by the obscured nucleus, as expected in the unified model of powerful radio sources (di Serego Alighieri, Cimatti \& Fosbury 1994). Spectropolarimetry is the most powerful technique to observe at the same time different radiation components, but the $4 \mathrm{~m}$ class telescopes can reach a sufficient $\mathrm{S} / \mathrm{N}$ ratio only on the few brightest objects. Therefore, in order to investigate the origin of the alignment effect and to test the validity of the unified model of powerful radio-loud AGN, we have started a pro- 
gram of optical spectropolarimetry of $\mathrm{H} z \mathrm{RG}$ s with the W.M. Keck $10 \mathrm{~m}$ telescope equipped with the Low Resolution Imaging Spectrometer (LRIS) in polarimetric mode.

\section{General results and implications}

We observed $6 \mathrm{H} z \mathrm{RG}$ s with $0.7<z<1.8$. Table 1 and Figure 1 show respectively the main results and one example of our spectropolarimetry. 3C 368 $(z=1.132)$ is not included in Table 1 because the data have been not fully reduced yet. - High linear polarization of the UV continuum is detected in all the observed galaxies (with the exception of $3 \mathrm{C} 356 \mathrm{~b}$ ). The perpendicularity of $\vec{E}$ to the UV continuum axis and the constancy of $\theta(\lambda)$ suggest that scattering is the dominant polarization mechanism. $\bullet$ The detection of the $\mathrm{MgII} \lambda 2800$ emission line in polarized flux in at least 3 galaxies suggests that the incident radiation comes from an obscured quasar nucleus and is emitted anisotropically along the radio axis. In particular, the broad and polarized MgII $\lambda 2800$ in 3C 324 has velocity and equivalent widths consistent with those observed in radio-loud quasars (Cimatti et al. 1995). On the other hand, the always lower or null polarization of the [OII] $\lambda 3727$ line implies that this line is emitted isotropically outside the obscuring region. $\bullet$ In the two galaxies analysed in detail so far (3C 256, Dey et al. 1995; 3C 324, Cimatti et al. 1995), we observe spatially extended polarization along the UV continuum axis, implying that the scattered flux is spatially extended. This result was possible thanks to the good seeing during the observations and the high $\mathrm{S} / \mathrm{N}$ ratio of our spectropolarimetry. - These results are in agreement with the requirement of the unified model of powerful radioloud AGN, where the differences between Type 1 and Type 2 AGN are mainly due to orientation effects and not to intrinsic diversities (Antonucci 1993). Our results also imply that scattered light is a necessary ingredient to explain the alignment effect. In particular, we find that scattered light contributes up to $50 \%$ of the total UV continuum in 3C 324 (Cimatti et al. 1995). - We detect basically two kinds of $P(\lambda)$ : flat and blue. Since the total flux spectra are generally red $\left(F_{\nu} \propto \nu^{-1 \div 2}\right)$, the polarized flux spectra $P \times F_{\nu}$ are either red or flat. This information will be used to investigate in detail the nature of the scattering particles, the incident spectrum and the importance of unpolarized UV radiation.

\section{References}

Antonucci, R., 1993, ARAA, 31, 473

Cimatti, A., Dey, A., van Breugel, W., Antonucci, R., Spinrad, H., 1995, ApJ, submitted Dey, A., Cimatti, A., van Breugel, W., Antonucci, R., Spinrad, H., 1995, ApJ, submitted di Serego Alighieri, S., Cimatti, A., Fosbury, R.A.E., 1994, $A p J, 431,123$

McCarthy, P.J., van Breugel, W.J.M., Spinrad, H., Djorgovski, S., 1987, $A p J, 321$, L 29 
TABLE 1. Summary of the results

\begin{tabular}{lcrrrrrr}
\hline Galaxy & $z$ & $\Delta \lambda_{\text {rest }}$ & $P_{\text {unb }}$ & $\vec{E}$ & $P(\lambda)$ & $\theta(\lambda)$ & $P \times F_{\text {tot }}$ \\
& & $(\AA)$ & $(\%)$ & & & & \\
& & & & & \\
3C 441 & 0.707 & $2350-5300$ & $2-10$ & $\perp$ & blue & const & const \\
3C 356a & 1.079 & $1900-4300$ & $7-15$ & $\perp$ & blue & const & const \\
3C 356b & 1.079 & $1900-4300$ & $2-4$ & $\perp$ & const? & const? & red? \\
3C 324 & 1.206 & $1800-4000$ & $10-13$ & $\perp$ & const & const & red \\
3C 13 & 1.351 & $1700-3800$ & $5-9$ & $\perp$ & const & const & red \\
3C 256 & 1.819 & $1400-3200$ & $10-14$ & $\perp$ & const & const & red \\
& & & & & & & \\
\hline
\end{tabular}

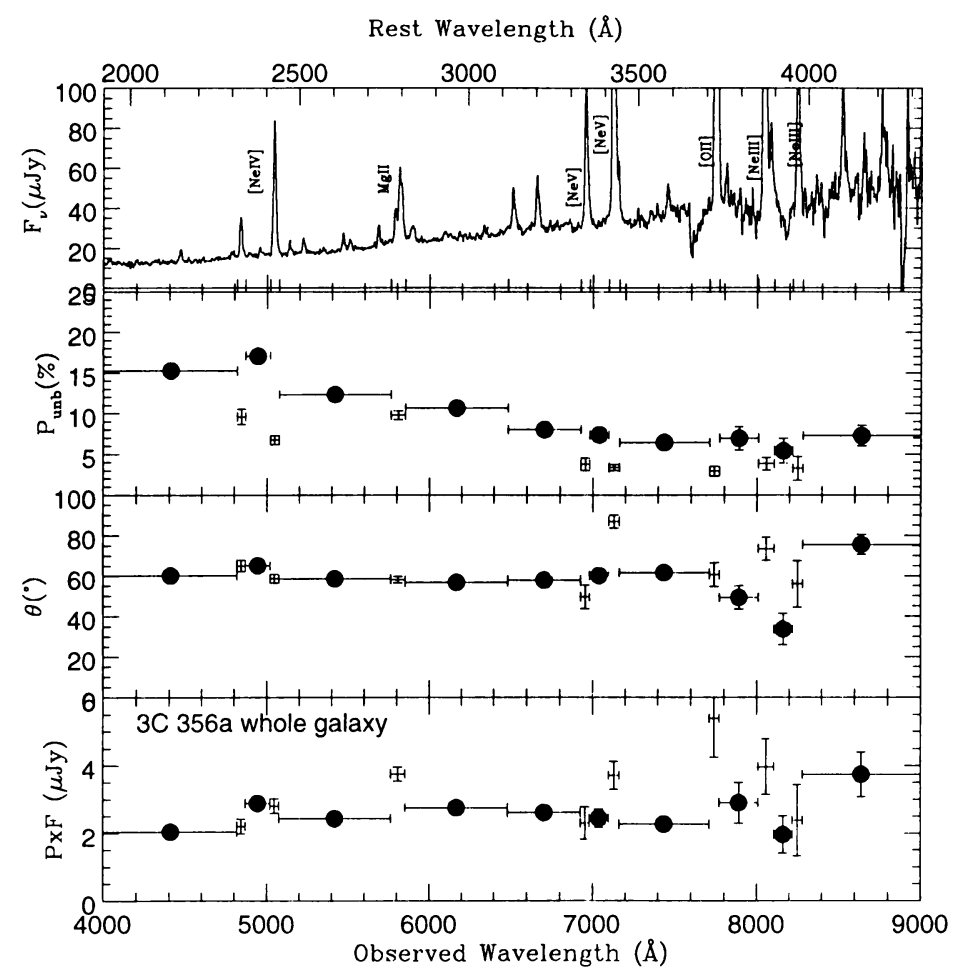

Figure 1. Keck spectropolarimetry of 3C 356a. From top to bottom : the total flux spectrum, the position angle of $\vec{E}$, the degree of polarization and the polarized spectrum. 